\title{
Central FPGA-based Destination and Load Control in the LHCb MHz Event Readout
}

\author{
Richard Jacobsson
}

\begin{abstract}
The readout strategy of the LHCb experiment [1] is based on complete event readout at $1 \mathrm{MHz}$ [2]. Over 300 sub-detector readout boards transmit event fragments at $1 \mathrm{MHz}$ over a commercial 70 Gigabyte/s switching network to a distributed event building and trigger processing farm with 1470 individual multi-core computer nodes [3]. In the original specifications, the readout was based on a pure push protocol. This paper describes the proposal, implementation, and experience of a powerful non-conventional mixture of a push and a pull protocol, akin to credit-based flow control. A high-speed FPGA-based central master module controls the event fragment packing in the readout boards, the assignment of the farm node destination for each event, and controls the farm load based on an asynchronous pull mechanism from each farm node. This dynamic readout scheme relies on generic event requests and the concept of node credit allowing load balancing and trigger rate regulation as a function of the global farm load. It also allows the vital task of fast central monitoring and automatic recovery in-flight of failing nodes while minimizing dead-time and event loss.
\end{abstract}

This paper demonstrates the strength and suitability of implementing this real-time task for a very large distributed system in an FPGA where no random delays are introduced, and where extreme reliability and accurate event accounting are fundamental requirements. It was in use during the entire commissioning phase of LHCb and has been in faultless operation during the first two years of physics luminosity data taking.

Keywords: Readout Control, Real Time Event Management, Load Balancing, Readout System, LHCb

\section{LHCb \\ THCP LHCb Public Note}

Issue:

LHCb-PUB-2011-023

Submitted to NIM A, December 7, 2011

Created: $\quad 30$ August 2005

Last modified: 24 November 2011

Prepared By R. Jacobsson, CEN, Geneva, Switzerland 



\section{Introduction and Requirements}

The LHCb experiment [1] is based on a single-arm forward spectrometer installed at one of the four interaction points on the LHC accelerator at CERN. It aims at studying CP-violation and rare decays with unprecedented precision by exploiting the large production of b-anti-b- and c-anti-cquark pairs at the LHC. The challenge of high trigger efficiency for many different hadronic final states has driven the choice of the readout architecture. Most importantly, the main event filtering is performed by a software High-Level Trigger (HLT) with access to all detector information on a very large processing farm based on commercial multi-core PCs. Consequently, LHCb has opted for a relatively simple first level trigger (L0) logic based on transverse energy and momentum of leptons, photons, and hadrons as measured by the calorimeters and the muon detector system, and full event readout to the Event Filter Farm at $1 \mathrm{MHz}^{1}$. The Event Filter Farm performs the main event selection with an accept rate of events to storage of about $3 \mathrm{kHz}$. The physics motivation and readout architecture put strong requirements on a flexible readout and trigger control with minimal dead-time and accurate event accounting.

The MHz readout architecture of LHCb is shown schematically in Figure 1 [4]. It consists of a set of detector-specific custom-made Front-End electronic boards connected to a set of 320 common Readout Boards [5] via approximately 5000 optical digital links and copper analogue links with a total data thru-put of the order of $4 \mathrm{~Tb} / \mathrm{s}$. The Readout Boards perform zero-suppression and interface the custom electronics to the Readout Network based on Gigabit Ethernet. Each Readout Board holds a fragment of an event. A router with the highest Gigabit Ethernet port density available currently ensures full connectivity with the processing farm and a bandwidth of $70 \mathrm{~GB} / \mathrm{s}$. The event building and HLT processing is performed by sending all fragments of the same event to the same node in the processing farm. In order to reduce the IP/Ethernet protocol overhead, event fragments from several consecutive events are packed together in Multi-Event Packets (MEP) [6]. Thus, the Readout Boards are required to assign the same IP destination to the MultiEvent Packets which contain the fragments of the same set of events.

In addition to the physics events, the experiment requires that the readout system is able to produce and cope with several different types of interleaved triggers for monitoring, calibration and accounting purposes, such as the readout of detector pulses, non-zero suppressed minimum bias events, beam-gas interactions, and luminosity triggers. Since some of these events require a different type of processing on a calibration farm, the system also has to cope with and synchronize properly the transmission to several destination types.

Rate regulation is required at many levels in the system, including the FE derandomizing buffers, the Readout Board processing and output bandwidth. All of these sources are accurately accounted for as dead-time. Apart from exceeding the nominal rate capacity of the processing farm due to statistical fluctuations in the rate or processing time per event, the readout network and processing farm, as well as the storage, may also temporarily suffer from a reduced rate capacity due to technical problems. As in any large processing system, individual nodes or set of nodes may also require re-initialization in-flight. In most standard data transmission protocols, all of these situations would normally lead to unaccounted event loss, and dead-time in recovering the system.

\footnotetext{
${ }^{1}$ The actually physical limit in readout rate from the Front-End electronics is slightly below $1.1 \mathrm{MHz}$.
} 
This paper addresses the requirements above and describes one of the aspects of the LHCb Timing and Fast Control (TFC) system [4][7] which deals with the management of the different types of events, the control of the event packing in the Readout Boards, the event destination assignment, and the load balancing and rate regulation of the processing farm. Section 2 briefly introduces the relevant aspects of the TFC system and the protocol implemented to control the data flow. Section 3 explains the destination assignment strategy and the load control, starting with the packing control in Section 3.1, followed by the event request mechanism in Section 3.2, the trigger rate control in Section 3.3 and finally the central monitoring of the distributed processing farm in Section 3.4. Section 4 describes the operational experience with the system during the two years of physics data taking and demonstrates the strength and the reliability of the solution.

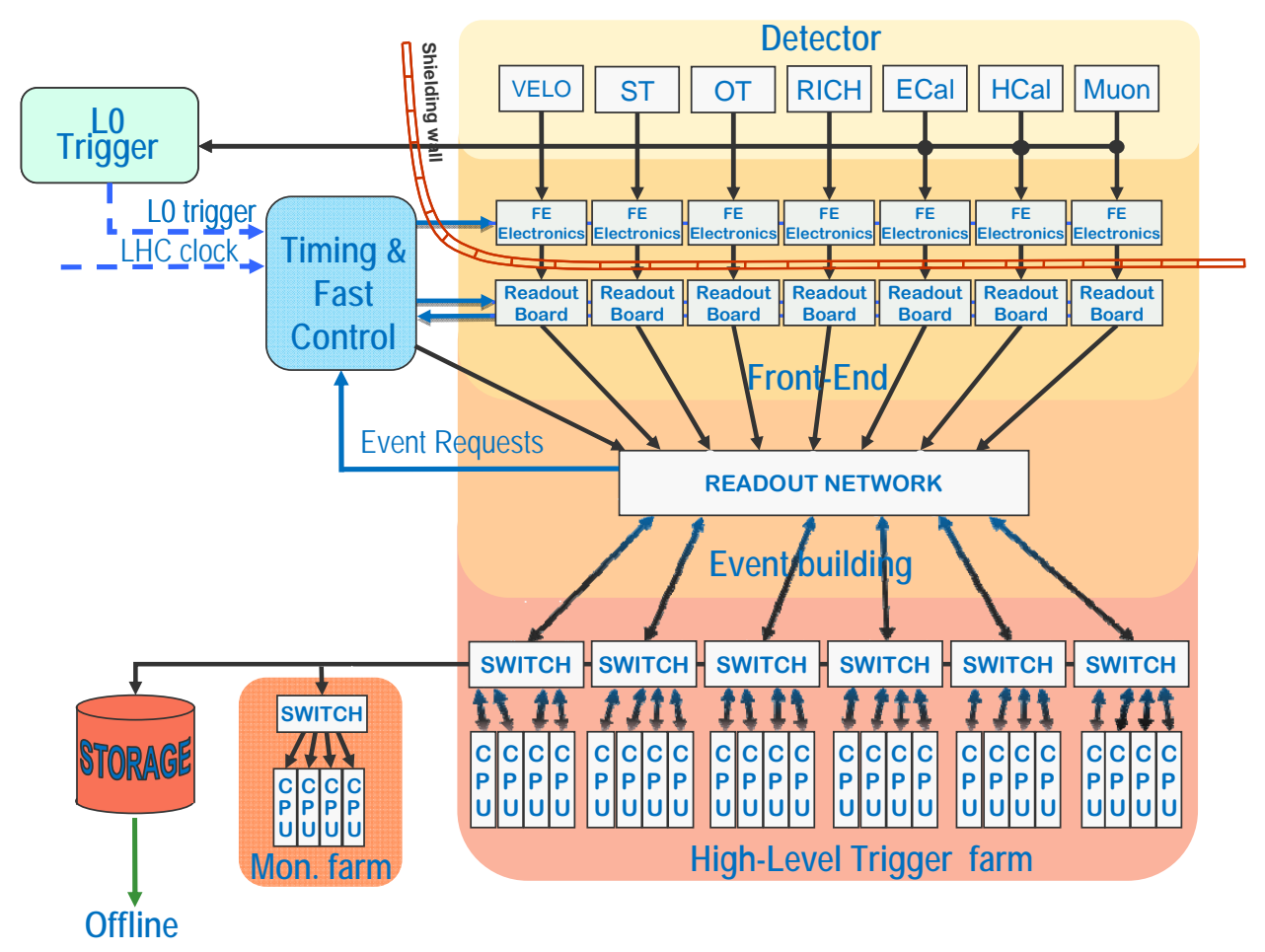

Figure 1: The architecture of the $\mathrm{LHCb} \mathrm{MHz}$ readout system. The TFC system performs the distribution of timing, the entire readout control, and event management. The CPU farm runs the event building and the event filtering from $1 \mathrm{MHz}$ to about $3 \mathrm{kHz}$ based on a software trigger.

\section{LHCb Readout and Trigger Control}

The timing, synchronization and control of the trigger and the entire dataflow from the Front-End electronics up to the Event Filter Farm are controlled by the LHCb Timing and Fast Control (TFC) system. It is entirely based on custom-made electronics, all of which are implemented with FPGAs. The system distributes the LHC bunch clock, trigger decisions, and synchronous as well as asynchronous commands to the electronics in the readout chain via a dedicated optical fibre network based on the CERN TTC common development [8]. The singular master of the TFC system is the Readout Supervisor (ODIN). A pool of optional and identical ODIN boards is connected to the sub-detectors via a configurable switch to allow parallel stand-alone runs of any subsystem. This also ensures the possibility of fail-over in case of failure of one ODIN during physics data taking. The L0 trigger decision unit is connected to the ODINs via a fan-out and deliver several types of decisions to ODIN. The ODIN internal logic performs the control of the 
readout synchronization and the trigger. It sequences internally the specific LHC bunch crossing scheme and, with the help of this information, generates random luminosity triggers and a wide variety of auto-triggers for calibration and test purposes. An event bank is prepared and transmitted over Gigabit Ethernet to the processing farm for every event like any Readout Board with information about the identity, time and source of the event. From this it follows that ODIN is also the main source of precise accounting of run statistics, luminosity, and dead-time.

The ODIN hardware was built with emphasis on flexibility and utmost reliability, and is organized around four large high-speed FPGAs for reasons of modularity. The FPGAs are connected together via a dense multi-purpose routing fabric. The logic implemented is developed in the VHDL language [9] and is operating with minimal latency for all functions in either the $40 \mathrm{MHz}$ domain of the LHC bunch clock or through internal PLLs at exactly double frequency. A four-port bi-directional Gigabit Ethernet mezzanine interfaces ODIN to the readout network and the processing farm. The control interface is based on a general-purpose control and monitoring protocol [10] implemented in a server with the Distributed Information Management (DIM) package [11] and running on an onboard credit-card-size PC [12][13]. The server communicates with the control system implemented with the SCADA tool PVSS from ETM [14].

The optical TTC network allows transmitting the LHC bunch clock to the readout electronics by adaptive clock recovery, and it provides two multiplexed serial data channels. The low latency channel at $40 \mathrm{Mbits} / \mathrm{s}$ is in LHCb used to distribute the L0 trigger decision. The second channel allows transmitting formatted data frames at $40 \mathrm{Mbits} / \mathrm{s}$ of two different types, referred to as short and long commands. The short command consists of eight user bits in a 16-bits word including Hamming code for data protection. The long commands consist of 16 user bits in 42-bits frames including the Hamming code.

In LHCb the low latency channel and the short commands are used to encode all of the information required to drive the readout of the Front-End electronics. The sections which follow will outline the details about how the short and the long commands are used to also control the event flow from the Readout Boards to the processing farm.

\section{Destination Assignment Strategy and Load Control}

Figure 2 shows a block diagram of the actual FPGA implementation of the destination control and the farm load control. The blocks are referred to in italics in the text.

\subsection{Multi-Event Packet control}

A scheme in which the control of the processing in the Readout boards and the assignment of the destinations for the Multi-Event Packets are handled centrally by ODIN has been implemented. The scheme has the advantage that the Destination Table, containing the IP addresses of the processing nodes, is stored in one location. It makes it straightforward to ensure the synchronicity of the event packing in the Readout Boards, and it imposes a certain level of concurrency in the readout. In addition, the implementation in ODIN contains a configurable real-time control of the processing type of the events in the Readout Boards and the destination type according to the trigger source. The Trigger Type Handler receives information about each trigger via the $L 0$ Accept FIFO from the LO Trigger Processing. The processing type is broadcasted for each trigger in the form of a short TTC command according to the format shown in Table 1. The two Least Significant Bits (LSB) of the L0 Event Identifier allow checking the synchronicity of the system. 
By construction the low latency of ODIN is such that the Trigger Type command always arrives to the Readout Boards with a maximum delay which guarantees no overflow at the input of the Readout Boards.

Table 1 : Trigger type command in which the trigger/processing type may be used to automatically decide on a different processing in the readout boards, e.g. non-zero suppressed/zero-suppressed etc.

\begin{tabular}{|c|c|c|c|c|c|c|}
\hline & 7 & 6 & 5 & 3 & 1 & $\mathbf{0}$ \\
\hline Trigger type command & 1 & & ocessing type & LSB LOEvID & 0 & 0 \\
\hline
\end{tabular}

Table 2: MEP Destination command in which the IP destination is encoded on twelve bits .

\begin{tabular}{|c|c|c|c|c|c|c|c|c|c|c|c|c|c|c|c|}
\hline & 15 & 14 & 13 & 12 & 11 & 10 & 9 & 8 & 7 & 6 & 5 & 4 & 3 & 2 & 10 \\
\hline IP destination & 1 & 0 & LSE & EvID & & & & & & $\mathrm{ad}$ & & & & & \\
\hline
\end{tabular}

The IP destination for each MEP is broadcasted by ODIN as a long TTC command according to the format shown in Table 2. Since all farm nodes have a common base address, only the two lower bytes of the IP addresses of the nodes are compressed in the 12-bit destination field. All other bits of the IP address are set by the base address configured directly in ODIN and in the Readout Boards via the control system. Again the two LSBs of the L0 Event Identifier allow checking the synchronicity of the Readout Boards.

The Event Packing Controller in ODIN controls the packet assembly in the Readout Boards of the event fragments into Multi-Event Packets by interleaving the Trigger Type commands with the Destination commands. The Trigger Type commands trigger the internal event processing in the Readout Boards and the Destination command triggers the closure of the MEP and the transmission to the processing farm. This allows a variable MEP packing factor to handle special triggers differently and close incomplete MEPs prematurely under special circumstances. Currently ODIN implements the following criteria to close MEPs automatically during a run:

- Configurable nominal packing factor which determines the maximum number of events in a MEP. This is the normal MEP size for a continuous run of any trigger type while the type is not changing.

- A change in the trigger type which is associated with a change in the destination type is immediately followed by a Destination command in order to close the current MEP and start filling a new MEP as long as the trigger type does not change again or the MEP packing factor is not reached. ODIN may also be configured to allow only one type of trigger per MEP. These mechanisms ensure that special triggers, such as for instance consecutive timing triggers or calibration triggers, may be sent to special farm destinations.

- If a MEP is still incomplete when ODIN starts an in-flight readout electronics reset sequence, a Destination command is sent in advance to flush the MEP.

- If the last MEP in a run is incomplete when the trigger is disabled by the control system, it is closed by an additional MEP destination issued by ODIN at the stop of triggers irrespective of the number of events in the open MEP.

- The sending of a MEP destination may also be triggered in-flight via the control system at any time as a single-shot action.

The Destination Table is configured by the Control System at the preparation for data taking. During running the destinations are retrieved by the Destination Controller from the Destination Table in a round-robin manner. The criterion for selecting a destination is explained in Section 3.2. 


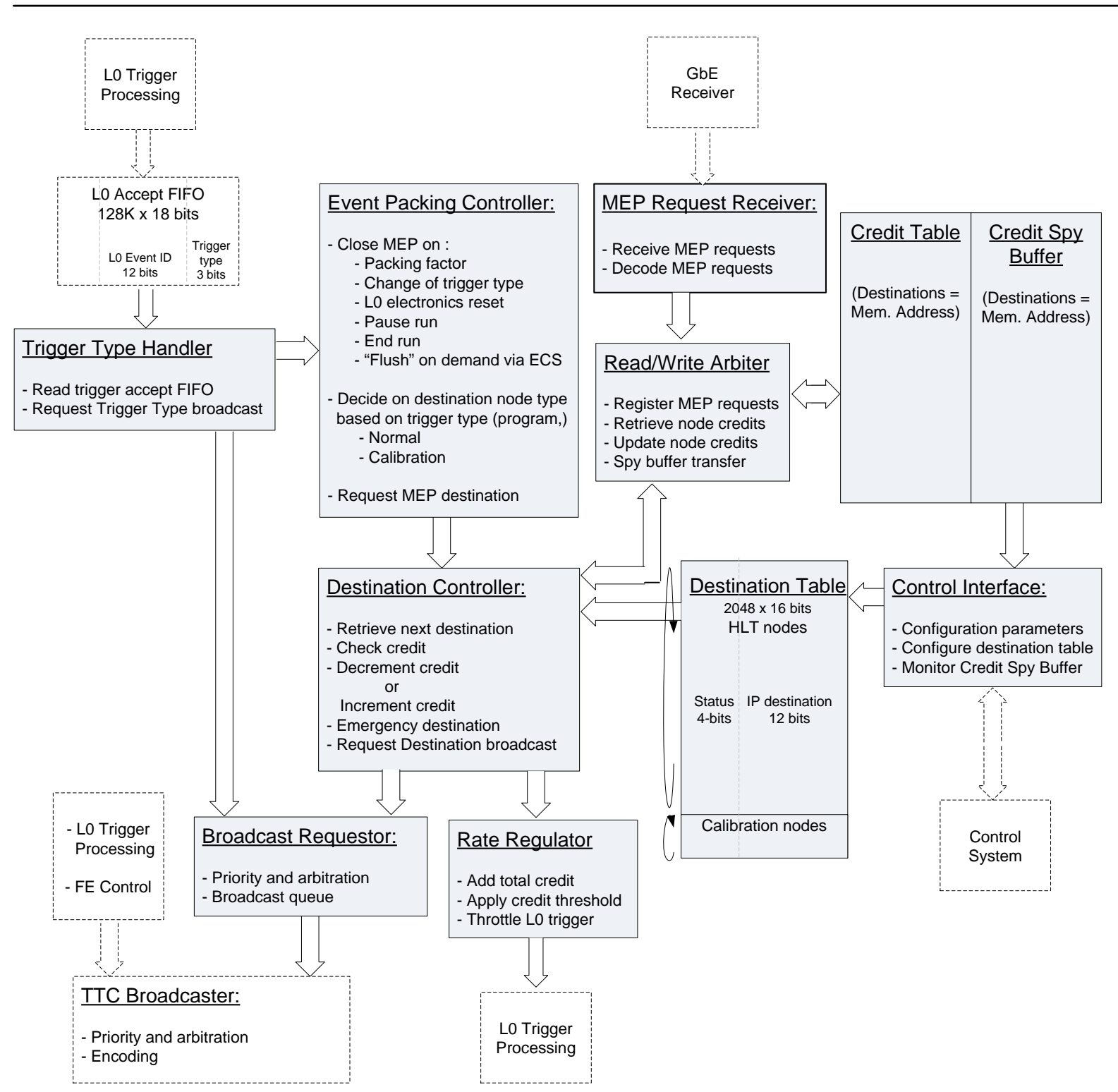

Figure 2: Block diagram of the VHDL implementation in the FPGA performing the dynamic destination assignment and load control scheme. The dashed white blocks represent logic located in other devices on ODIN.

Consequently, the scheme does not perform a dynamic balancing of the load and traffic on the readout network since the order in which the destinations are retrieved is fixed by the destination table. However, a fixed load balancing is obtained by ordering the destinations in the table in a manner which takes into account the topology of the network and the organisation of the farm nodes with different processing capacity. For instance, in order to equilibrate the load distribution on the network switch, the destinations in the table are organized such that the output links towards a set of farm nodes which is covered by a single sub-unit in the network switch with shared resources is not addressed twice in a row. This is illustrated by the internal subdivision of the readout switch and the arrows in Figure 3.

Since ODIN is designed to handle different types of destinations, each of the trigger types may be associated with a specific type of destination. As shown in Figure 2, currently ODIN holds separate tables for the normal event filter farm with up to 2048 nodes and for a calibration farm with up to eight nodes. 
In practice the destinations are pre-fetched meaning that as soon as the Destination Controller is enabled, it will immediately retrieve a destination waiting for triggers and the first request for MEP closure from the Event Packing Controller. On every L0 trigger accept, the Trigger Type Handler receives source information about the trigger and its processing type via a derandomizing LO Accept FIFO. It then prepares the Trigger Type command, stores it in a broadcast queue in the Broadcast Requestor for Trigger Type commands and Destination commands and submits a transmission request to the TTC Broadcaster. The queue allows the Trigger Type command to stay pending in case the TTC Broadcaster is busy with another transmission or a clash with another type of command of higher priority.

Upon a closure of a MEP by either a demand via the control system or due to any of the reasons above, the Destination Controller adds a destination header to the pre-fetched destination and writes the Destination command to the broadcast queue mentioned above. At the same time, the Broadcast Requestor submits a transmission request to the TTC Broadcaster. The Event Packing Controller will immediately reset the MEP event counter, while the Destination Controller prefetches the next destination irrespectively of whether another trigger arrives or not.

It follows from the $40 \mathrm{Mbits} / \mathrm{s}$ bandwidth of the command channel and the size of the short (16 bits) and the long command (42 bits) that the minimum packing factor at $1 \mathrm{MHz}$ is three. At normal running the nominal packing factor is typically configured to be between 10 and 15 . A packing factor of one is only used for tests below a L0 trigger rate of $\sim 650 \mathrm{kHz}$.

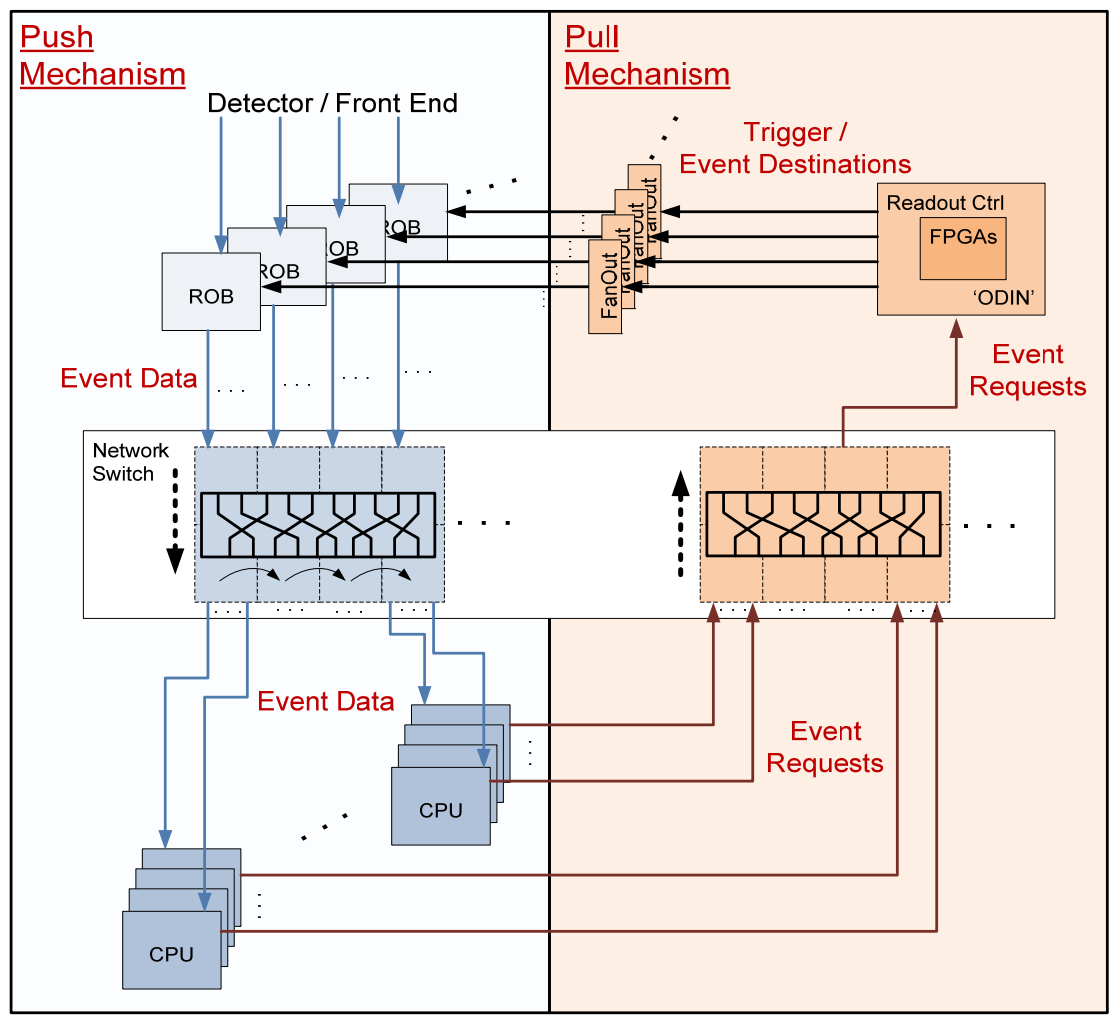

Figure 3: Logical diagram illustrating the mixture of a push protocol and a pull protocol akin to a credit-based flow control as handled by the central FPGA-based readout controller. The packing and the transmission of the events by the Readout Boards are driven by trigger and destination commands from the controller. The artificial split between down/up direction in network the switch is only to illustrate more clearly the functionality of the two directions. In reality the system consists of 320 Readout Boards and 1470 CPU nodes. 


\subsection{Dynamic Event Destination Assignment}

As shown in Figure 3, adding a mechanism by which each farm node declares itself as available for processing an event by sending 'Event Requests' to ODIN introduces a powerful load control mechanism which may account for both different CPU performances and statistical fluctuations in the processing times. More importantly, it introduces a dynamic mechanism which copes with node failures and node recovery of any type with minimal event loss. Effectively, ODIN selects the MEP destinations in the Destination Table according to the availability of the farm nodes. This leads to a completely auto-regulated system. In addition, the event requests are generic requests and do not refer to a particular event, which makes the system robust against possible request packet losses.

In order to take into account the latency in the readout network and not to starve the multi-core CPUs, each MEP must contain up to 10-15 events. In addition, the implementation of the logic in ODIN allows each node to request several MEPs at a time by storing a "credit" for each node. Currently, the initial MEP Requests at the start of data taking carry a credit of three MEPs, while all subsequent MEP Requests during data taking carry a credit of one MEP at a time.

In practice, the Multi-Event Packet Requests are sent by the Event Builder tasks [15] running in the farm nodes. The MEP Request packets are standard minimum-sized IP packets with a special Protocol Type and with only the Destination Credit as the first word in the payload.

ODIN receives the MEP Requests directly via Gigabit Ethernet. The ODIN MEP Request Receiver is able to receive and decode the MEP Requests at Gigabit Ethernet wire-speed, and add the Destination Credit in the Credit Table. This effectively means a MEP Request rate of over 1.8 $\mathrm{MHz}$ and thus copes safely with the start of the data taking phase when all the 1470 farm nodes declare themselves to ODIN within a very short period of time. No losses of request packets have been observed in ODIN.

The Credit Table in ODIN is eight bits wide and contains 4096 memory locations corresponding to the 12-bits encoded IP address of the farm nodes. Thus, for speed, the IP source address is used by ODIN directly to address the credit for each node in the Credit Table. The Destination Controller loops over the destinations in the Destination Table and, using the IP address, looks up the credit for each node in the Credit Table. It selects the first destination it finds with a positive credit and decrements the credit value by one. The 8-bit values are signed such that the Destination Controller decrements the credit below zero when it passes a destination which have not requested a MEP since the last pass through the Destination Table. Since processing times of the MEPs in the farm nodes vary statistically, it is normal to have nodes with a distribution of negative credits. Effectively, it is a measure of the load on the farm and "-127" indicates that the farm node is permanently out of order. A spying mechanism explained below allows monitoring the Credit Table in-flight.

An Arbiter logic manages the access priority to the Credit Table between the MEP Request Receiver, the Destination Controller, and the spy mechanism. The MEP Request Receiver has the highest priority and increments the credit for a destination in a two-clock read-add-write action using the IP Source Address of the IP packet as memory address in the Credit Table. In case the particular destination has a negative credit because it has been unavailable for more than one pass of the Destination Controller, the negative credit is cleared and the credit in the Request is registered. The spy mechanism operates at "best effort" only and copies the Credit Table contents in idle clock cycles when it is not accessed by neither the MEP Request Receiver, nor the Destination Controller. 
Normally with an event packing factor of 10 at $1 \mathrm{MHz}$, the destination search algorithm is able to lookup the credit for over 100 destinations before a destination must be available for broadcasting to avoid the risk of overflow in the output buffer of the Readout Boards. Even with the minimum packing factor of three at $1 \mathrm{MHz}$, the algorithm is able to check on 50 destinations in the table. If, for technical reasons, a destination with a positive credit has not been found in the maximum time allowed, a safeguard timeout mechanism forces an emergency destination to be used to close the Multi-Event Packet to continue the run smoothly without dead-time. An error counter logs these cases. By construction, only exceptional circumstances would lead to such a situation (partial power cuts, incorrect handling of exceptions in the HLT processing etc).

A conventional pull protocol, in which the requests refer to specific sets of data, risks blockages if a request gets lost unless it has retransmission mechanism, which in itself adds a tail of increased latency and increased demand on buffering. In the current system, losses of event requests are rapidly diagnosed through the spy mechanism, which allows recovering the missed-out nodes through the control system before it has a significant impact on the rate capacity of the farm. In all cases, the system allows the critical task of maintaining precise accounting of the dead-time and recorded luminosity of the experiment.

For specific tests, the dynamic assignment based on the node credit may be disabled turning it into a normal static destination assignment without trigger rate control.

\subsection{Trigger Rate Regulation Mechanism}

The total processing capacity, i.e. event rate capacity of the farm may be affected for several reasons. There may be failing nodes, processes stuck, excessively long processing times due to events with large complexity, excessively noisy background conditions, or failures in the network, or even back pressure from the storage if the throughput limit to disk storage is exceeded. The MEP Request scheme allows implementing a central trigger throttle mechanism which effectively regulates the rate of the L0 trigger to the current capacity of the farm and the storage. This reduction in L0 trigger rate is accurately accounted for as dead-time. It is typically a transient situation and the mechanism controls and minimizes unaccounted event loss. In addition, in many cases it prevents the situation from getting worse and thus also maximizes the data-taking efficiency.

The rate regulation is based on the total credit available. The Rate Regulator in ODIN maintains a sum of the total credit and applies a configurable threshold, which if exceeded will transmit a trigger rejection signal to the LO Trigger Processing. In terms of the L0 rate, the system has a very sharp saturation threshold by construction as discussed in Section 4. This, together with the short latencies and the fast search over many destinations in between Multi-Event Packets, implies that the total credit limit may be set very low. Experience shows that a threshold of 5-10\% of the total credit is appropriate taking into account that each node registers a credit of three at initialization.

The trigger rate regulation mechanism will also handle properly the case when a set of farm node has to be intervened on and recovered. The reduced farm capacity will be properly accounted for by reducing the L0 trigger rate, effectively producing temporary dead-time. As soon as the nodes are initialized, they automatically transmit an event request and thus get automatically included in the data taking again in-flight.

The throttle mechanism may be enabled or disabled. If the MEP Request handling and the throttle 
mechanism are disabled, the scheme continues to work and performs as a round-robin destination assignment with only static load balancing of the readout network. The MEP destinations will assume a 'random' order since in this particular case the search for available destination continues and the actual destination chosen for each MEP depends on the time between triggers.

\subsection{Central Monitoring of the Farm Nodes and Recovery}

A spy mechanism allows getting a complete dump of the Credit Table through the Credit Spy Buffer. The mechanism is triggered via the control system and the Arbiter copies the Credit Table to the Credit Spy Buffer on idle cycles while it is not accessed by the MEP Request Receiver or the Destination Controller. This means that it is not an instantaneous snapshot of the table but typically read over a period of about $10 \mathrm{~ms}$ while the trigger rate is $1 \mathrm{MHz}$. However, this is sufficient for the two main purposes of the information, the first of which is to know the distribution of negative credits (see Section 3.2) to monitor the global load on the farm, and the second of which is to know the nodes which have a credit of "-127" and thus require recovery actions by the control system. A status bit is set as soon as the dump is complete. This triggers the readout by the control system. Typically the complete dump is produced every 30 s.

Thus the scheme has the advantage that it allows rapid monitoring of the individual farm nodes in a large distributed system. The control system may then be automatically triggered to perform a reset, or even a reboot of the nodes, and re-initialization. As the nodes transmit an event request on initialization, they automatically get included again in the data taking.

\section{Operational Experience}

The logic presented in this paper was implemented in 2006 and was in operation during the entire commissioning phase of LHCb and the two years of physics data taking 2010-2011. During 2011 LHCb reached close to its nominal L0 rate of $1 \mathrm{MHz}$. However, due to very successful design of the detectors and the readout system, LHCb managed during these years to operate the experiment at a luminosity well above its design, and reach in 2011 stable running at an average luminosity of $4 \times 10^{32} \mathrm{~cm}^{-2} \mathrm{~s}^{-1}$. This is equivalent to twice the design luminosity. While the maximum L0 rate of one $\mathrm{MHz}$ is a design limit in the Front-End electronics, the success of running at higher luminosity was achieved by increasing the luminosity per colliding bunch pair, i.e. by taking data with four times the design event pile-up. This in itself was possible thanks to a major effort to increase the bandwidth through the Readout Boards and the Readout Network, an important increase of the farm size, a very well optimized High-Level Trigger, and an output bandwidth to storage of close to $800 \mathrm{MB} / \mathrm{s}$ allowing readout of more than $3 \mathrm{kHz}$ of events. In addition, LHCb has implemented together with the LHC machine a mechanism by which the luminosity may be controlled by semicontinuously adjusting an head-on offset between the beam axes of the colliding bunches [XX]. This allows levelling the LHCb luminosity to an almost constant value throughout the LHC fills.

In these conditions, the MEP Request Receiver has been handling MEP Requests from 1470 distributed farm nodes. With a nominal MEP packing factor of 13, the MEP Request Receiver and the Destination Controller perform the destination assignments at an average rate of about 70 $\mathrm{kHz}$. No MEP Requests were ever lost in ODIN during the peak loads of up to a $1.8 \mathrm{MHz}$ at the start of data taking when all nodes transmit the initial MEP Request during a very short period of time. During data taking, the system is normally handling five different types of triggers to two different types of destinations. 


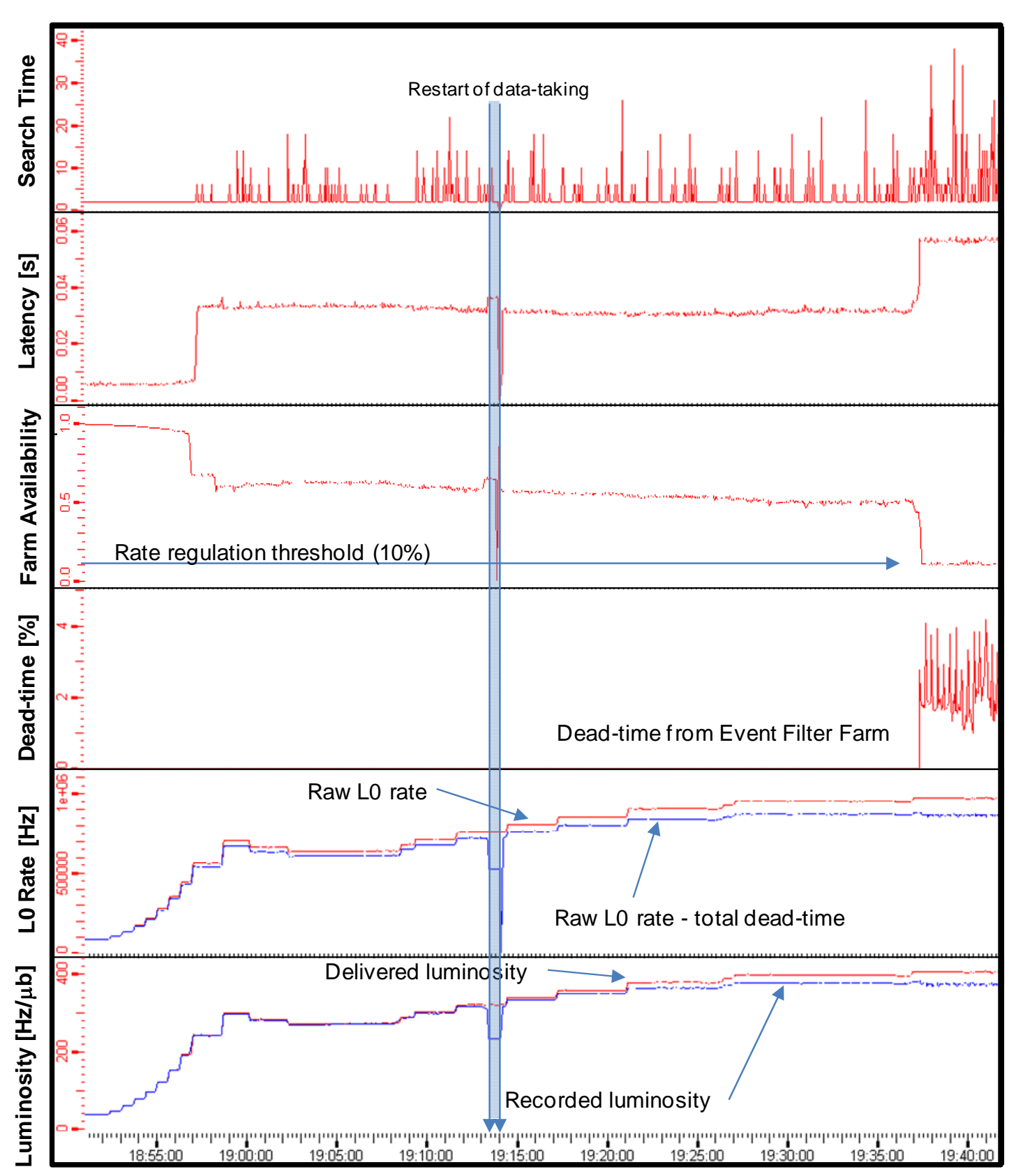

Figure 4: Trend plot showing the main system parameters during the first hour of a physics fill while the luminosity is ramped up to $4 \times 10^{32} \mathrm{~cm}^{-2} \mathrm{~s}^{-1}$. In this example, the luminosity is ramped to slightly above $4 \times 10^{32} \mathrm{~cm}^{-2} \mathrm{~s}^{-1}$ which exceeded the current processing capacity of the farm. As a result the load control automatically reduces the LO trigger rate which leads to a dead-time of about $2.5 \%$.

Figure 4 shows a trend plot of the main system parameters during the first hour of a physics fill while the luminosity was being ramped from about $0.4 \times 10^{32} \mathrm{~cm}^{-2} \mathrm{~s}^{-1}$ up to running flat at about $4 \times 10^{32} \mathrm{~cm}^{-2} \mathrm{~s}^{-1}$. In addition to the Delivered and the Recorded Luminosity, the plot shows the raw input LO Trigger Rate and the rate which is accepted after dead-time subtraction. There are in reality several sources of dead-time which currently add up to about $5.5 \%$ at $4 \times 10^{32} \mathrm{~cm}^{-2} \mathrm{~s}^{-1}$. The Dead-time parameter shown in the trend plot is purely dead-time introduced by a saturation of the processing farm as regulated by the load control mechanism. The Farm Availability is the fraction of the processing farm which is available for receiving events. The Latency is a measure of the 
average total round-trip time from the moment a MEP destination is transmitted by ODIN to the Readout Boards, including the MEP closure, up to the point that the MEP Request from that destination is received by ODIN again. The Search Time is a measure of the number of destination that ODIN checks before finding a destination which is available for receiving a MEP. Since the destination search requires two clock cycles per destination, the value corresponds to the number of address pointer shifts times two.

As seen from the farm availability and the latency in Figure 4, the system exhibit two threshold effects. Up to a L0 trigger rate of about $570 \mathrm{kHz}$, which corresponds to a destination assignment and a MEP request rate of about $44 \mathrm{kHz}$, the latency is about $6-7 \mathrm{~ms}$ and the farm availability constantly above $90 \%$. Since an idle farm node essentially requests the next MEP as soon as it receives a MEP and starts processing immediately, the $6-7 \mathrm{~ms}$ corresponds to a latency offset coming from the minimum round-trip time. This may also be seen from the search time which shows that the next node in the table is always available.
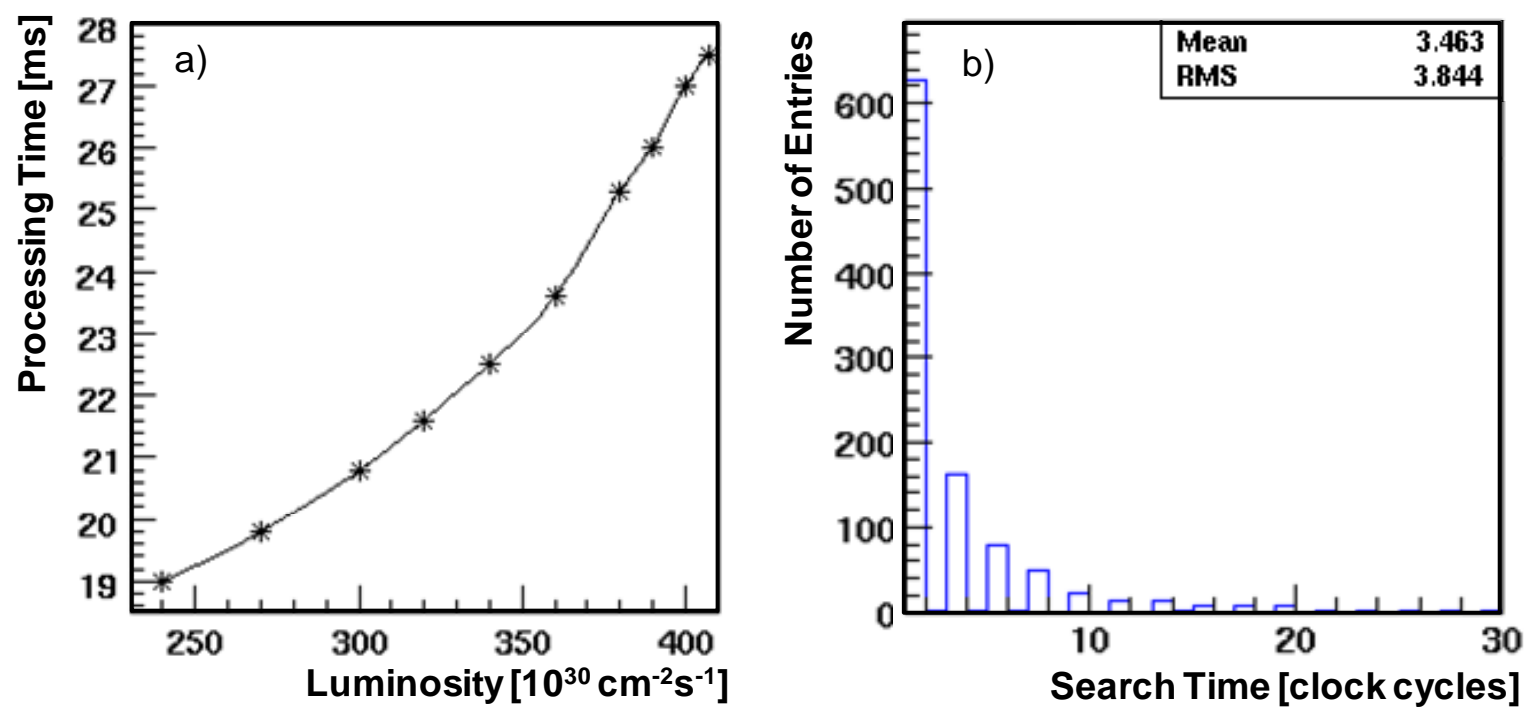

Figure 5: The left plot a) shows the event processing time by the High Level Trigger as a function of the luminosity. It is due to the increasing event pileup as the luminosity per colliding bunch pair is increased. The right plot b) shows the search time in terms of clock cycles for a free destination while the farm is running at the limit of its capacity. First bin corresponds to the next destination in the table being available. Retrieving subsequent destinations require two clock cycles each.

At a MEP rate of $44 \mathrm{kHz}$ to the 1470 farm nodes, each node receives a MEP for processing every $\Delta \mathrm{t}_{\mathrm{MEP}} /$ node $\sim 30 \mathrm{~ms}$. Figure $5 \mathrm{a}$ shows the High Level Trigger processing time per event as a function of the luminosity. The average processing time per event at this luminosity is about 20ms and the events in a MEP are processed in parallel on the multi-core nodes. This means that the average $t_{\text {tranmission }}($ MEP $)+t_{\text {processing }}($ MEP $)+t_{\text {Request }}(M E P) \sim \Delta t_{\text {MEP }} /$ node. As this rate is exceeded, the statistical spread in processing time, the nominal packing factor of 13 , and the number of processing tasks running in parallel on each node (average 17), leads to the farm becoming busy to about $50 \%$. As the luminosity increases and consequently the L0 trigger rate increases the farm becomes increasingly busy linearly up to a rate of $920 \mathrm{kHz}$. The linear increase in the farm occupancy also means that the latency remains constant at about $31 \mathrm{~ms}$ corresponding to approximately the $t_{\text {tranmission }}(\mathrm{MEP})+t_{\text {processing }}(\mathrm{MEP})+t_{\text {Request }}(\mathrm{MEP})$. As a second consequence, the statistical variations in the event processing time and the increasing average processing time from $20 \mathrm{~ms}$ to $27 \mathrm{~ms}$ at close to $4 \times 10^{32} \mathrm{~cm}^{-2} \mathrm{~s}^{-1}$, also implies that nodes are occasionally still busy even after a full turn of the destination table. This is seen by the increasing search time for the next 
destination. Care has to be taken to adjust the system parameters such that this effect does not prevent the system from exploring the full processing power since if a node is busy, ODIN will only return to this address after a full turn of the destination table. The fast search allowing the algorithm to lookup over 100 destinations in between MEPs, the large packing factor, and the low threshold for the rate regulation allows minimizing this effect.

At a rate of $920 \mathrm{kHz}$, the MEP rate is about $71 \mathrm{kHz}$ meaning that each node receives a MEP every $21 \mathrm{~ms}$. This is faster than the average processing time of $27 \mathrm{~ms}$ and should lead to overcommitment. However, since the farm nodes run on the average 17 processing tasks and the packing factor is 13, it allows coping with a another 30\% of MEP rate. This has also been confirmed by monitoring the CPU load on the nodes to verify that it reaches close to $100 \%$.

Beyond $71 \mathrm{kHz}$, the farm quickly reaches saturation and the availability falls rapidly to $10 \%$ which is the threshold used to regulate the rate. Figure 4 shows the dead-time increasing to about $2.5 \%$ which then forces the rate to remain at about $920 \mathrm{kHz}$. During saturation, the loss of MEPs due to a lack of a free destination remains well below $10^{-7}$. Figure $5 \mathrm{~b}$ shows the Search Time distribution while running at the limit of the system.

It should be noted that the scheme does not aim at performing balancing of the CPU load on the different nodes. A less performing node will be busier than a fast node. However, experience shows that this has no impact on the system performance since the algorithm is ultra-fast and is able to search over a large number of destinations between completions of MEPs. The system is able to utilize the full capacity of the fast nodes without dead-time and degradation in the performance. Nor does it aim at shaping the traffic on the network except for what is achieved with the static organization of the destinations in the table, which takes into account the network topology and avoids overloading the switch output links which share internal resources.

\section{Conclusion}

In the LHCb experiment, an ultra-fast FPGA-based central system, synchronous with the LHC accelerator, performs the entire synchronization, event labelling and control of the readout from the Front-End electronics up to the Event Filter Farm. The system is driven by a single master, the Readout Supervisor (ODIN), but incorporates a fail-over mechanism for fault tolerance. The readout network and the Event Filter Farm is a very large distributed system of 1470 processing nodes which receives about one $\mathrm{MHz}$ of events from 320 Readout Boards in the form of event fragments packed into Multi-Event Packets.

This note describes the proposal and the implementation of a dynamic event management scheme which performs the event destination assignment and the load control of the readout network and the farm in a manner akin to a credit-based flow control. The control logic is implemented in the ODIN and is based on a central handling of a pull protocol consisting of asynchronous and generic event requests from each processing node. The implementation allows a dynamic control of the processing type of the events and allows controlling the number of events which is packed into each Multi-Event Packet. The scheme leads to a completely auto-regulated and flexible system which is robust against losses of processing nodes and which guarantees minimum unaccounted event loss. A central and fast monitoring of the state of each farm node allows rapid diagnostics and recovery in-flight of failing nodes. Moreover, as the nodes get included automatically by their event requests on initialization, the operational efficiency was improved significantly by starting data taking already when a majority of the farm is ready for data taking instead of waiting until the last node is configured. 
The choice of an FPGA based implementation has proven extremely reliable. The readout control and event management has performed faultlessly over four years contributing significantly to a total operational efficiency of LHCb of well over 90\% during the first two years of data taking, during which LHCb succeeding in running the experiment at twice its design luminosity.

The flexibility of the FPGA-based solution would allow integrating more advanced search algorithms if it proves necessary to explore fully the capacity of the farm, and it has been proposed for the upgrade of LHCb [16][17].

\section{Acknowledgements}

This work was done in the context of the LHCb Collaboration. I would like to thank my colleagues Jean-Pierre Dufey, Markus Frank, Beat Jost, and Niko Neufeld for many fruitful discussions and suggestions. I would like to thank Federico Alessio for encouraging me to write this paper and for proof-reading.

\section{References}

[1] The LHCb Collaboration, "The LHCb Detector at the LHC", 2008 JINST 3 S08005.

[2] P.R. Barbosa-Marinho et al., "LHCb Online system technical design report: Data acquisition and experiment control”, CERN-LHCC-2001-040

[3] M. Frank et al., "The LHCb high level trigger infrastructure,” in J. Phys Conf. Ser., 2008, vol. 119, p. 022023.

[4] F. Alessio et al., "The LHCb readout system and real-time event management”, IEEE Trans. Nucl. Sci., vol. 57 (2010) 663.

[5] G. Hafeli, et al., “The LHCb DAQ interface board TELL1”, Nucl. Instrum. and Meth. A 560 (2006).

[6] LHCb Collaboration, “Addendum to the LHCb Online System Technical Design Report”, CERNLHCC-2005-039.

[7] Z. Guzik, R. Jacobsson, B. Jost, "Driving the LHCb Front-End Readout”, IEEE Trans. Nucl. Sci., vol. 51 (2004) 508.

[8] B. G. Taylor, “TTC distribution for LHC detectors,” IEEE Trans. Nucl. Sci., vol. 45, no. 3 (1998) 821.

[9] IEEE VHDL Analysis and Standardization Group, [online] http://www.eda.org/vasg/

[10] R. Jacobsson, "Building Integrated Remote Control Systems for Electronics Boards”, IEEE Trans. Nucl. Sci., vol. 55, no. 1 (2008) 336.

[11] C. Gaspar, Distributed Information Management System, [online] http://cern.ch/dim/

[12] F. Fontanelli et al., "Embedded Controllers for Local Board Control”, IEEE Trans. Nucl. Sci., vol. 53 (2005) 936.

[13] Z. Guzik and R. Jacobsson, "Glue Light”- A Simple Programmable Interface between the Credit Card PC and Board Electronics”, LHCb Tech. Note 2003-56, 2003.

[14] ETM Professional Control, [online] http://www.pvss.com/

[15] Neufeld, et al., “The LHCb event-builder”, IEEE Trans. Nucl. Sci., vol. 58, no. 4 (2011)1877.

[16] F. Alessio, Z. Guzik, R. Jacobsson, "Timing and Fast Control and readout electronics Aspects of the LHCb Upgrade”, LHCb-PUB-2008-072.

[17] F. Alessio, Z. Guzik, R. Jacobsson, “A $40 \mathrm{MHz}$ Trigger-free Readout Architecture for the LHCb Experiment”, proceedings of IEEE Real Time Conference 2009, Beijing, China. 